\title{
The Effects of Radial Compression on Thermal Conductivity of Carbon and Boron Nitride Nanotubes
}

\author{
Haijun Shen \\ School of Aerospace and Mechanics, Tongji University, Shanghai 200092, China \\ Correspondence should be addressed to Haijun Shen, shj@tongji.edu.cn \\ Received 20 September 2011; Accepted 30 January 2012 \\ Academic Editor: Teng Li
}

Copyright () 2012 Haijun Shen. This is an open access article distributed under the Creative Commons Attribution License, which permits unrestricted use, distribution, and reproduction in any medium, provided the original work is properly cited.

By using molecular dynamics method, thermal conductivity of $(10,10)$ carbon and boron nitride (BN) nanotubes under radial compression was investigated, and the $\lambda-T$ (thermal conductivity versus temperature) curves of the two nanotubes were obtained. It is found that with the increase of temperature the thermal conductivity of two nanotubes decreases; the nanotubes, under both the local compression and whole compression, have lower thermal conductivity, and the larger the compressive deformation is, the lower the thermal conductivity is; the whole compression has more remarkable effect on thermal conductivity than the local compression.

\section{Introduction}

As the feature sizes of MEMSs (microelectro mechanical systems) continue to be scaled down, the problem of thermal dissipation becomes even more important. It has been found that very few materials are known to exhibit high thermal conductivity at reduced dimensions, and traditional semiconductors like Si have a dramatic suppression of thermal conductivity at the nanoscale [1].

Just like carbon nanotubes can be viewed as rolling a graphite sheet into a cylinder, boron nitride (BN) tubes can also be regarded as geometrically derived by rolling a hexagonal BN sheet into a cylinder. Due to low-dimensional effects, carbon and BN nanotubes have been predicted to have unusual thermal properties $[2,3]$. The thermal conductivity of an isolated carbon nanotube has been predicted to reach $1800-6000 \mathrm{~W} / \mathrm{mK}$ at room temperature [4]. Similarly, the thermal conductivity $(290 \mathrm{~K})$ of isolated $\mathrm{BN}$ nanotube has been reported to be $1620 \mathrm{~W} / \mathrm{mK}$ [5].

Recent $\mathrm{Xu}$ and Buehler [6] carried out a systematic investigation of the effects of axial tensile, compressive and torsional strain on the thermal conductivity of single-walled carbon nanotubes using MD simulation, and found that the thermal conductivity drops when the different strain is applied to carbon nanotube, respectively. However, up to now the work about the effects of radial compression on carbon nanotube as well as $\mathrm{BN}$ nanotube is not found. Here we perform nonequilibrium MD simulation to investigate the thermal conductivity of the $(10,10)$ carbon and $\mathrm{BN}$ nanotubes under radial compression and discuss the effects of the local and whole compressive deformation on thermal conductivity of the carbon and $\mathrm{BN}$ nanotubes.

\section{The Investigated Objects and Method}

2.1. The Investigated Objects. Figure 1 shows the investigated objects, $(10,10)$ zigzag carbon and $\mathrm{BN}$ nanotubes. Considering that within the length range of $1 \mathrm{~nm}$ to $25 \mathrm{~nm}$ nanotubes have little change in thermal conductivity [7], the present tube length is taken to be $8.85 \mathrm{~nm}$ to $9.81 \mathrm{~nm}$. The $(10,10)$ carbon tube is generated by the nanotube generator [8], a program module developed by us. The $(10,10) \mathrm{BN}$ tube is constructed by alternately replacing the carbon atoms of $(10,10)$ carbon tube with boron or nitrogen atoms. After geometrically optimizing the carbon and $\mathrm{BN}$ nanotubes through MD simulation at $270 \mathrm{~K}$, the initial configurations are obtained. The initial diameter $D$ of the carbon and $\mathrm{BN}$ nanotubes is $1.36 \mathrm{~nm}$ and $1.52 \mathrm{~nm}$, respectively.

2.2. Method. In classical MD, atoms are regarded as basic particles, and the temperature of the simulated system is given through the equipartition theorem of energy. When the 
practical temperature is lower than the Debye temperature of material, the thermal capacity will be highly correlative with the temperature of system, the equipartition theorem of energy will become invalidated [9], and the quantum correction to temperature must be performed. In the present MD calculations of thermal conductivity, the temperature of both the nanotube systems is taken above their Debye temperature so that quantum effects can be ignored. The Debye temperature of the carbon and BN nanotubes takes $420 \mathrm{~K}$ [10] and $400 \mathrm{~K}$ [11], respectively.

In this study the EMD (equilibrium molecular dynamics) method with the time step of $0.5 \mathrm{fs}$ is applied to geometrically optimize the carbon and $\mathrm{BN}$ nanotubes, as well as to calculate the thermal conductivity of the carbon and $\mathrm{BN}$ nanotubes locally or wholly compressed in radial direction (see Figure 1). The process to calculate the thermal conductivity can be described as the following: firstly relaxing the tubes in the NTV ensemble for $3 \times 10^{5}$ time-steps, then locally or wholly compressing the carbon and $\mathrm{BN}$ nanotubes in the radial direction through two parallel rigid plates, where the loading rate takes $0.03 \mathrm{~nm}$ per 1000 time steps, and the temperature is controlled at $270 \mathrm{~K}$ through Nose method; fixing the two rigid plates when the carbon and $\mathrm{BN}$ tubes reach certain deformation, calculating the heat flux of each time step for the subsequent $4 \times 10^{6}$ time-steps, and obtaining the thermal conductivity of the compressed tubes through integrating the autocorrelation function of heat flux [12].

In the present MD simulations, the Velocity-Verlet algorithm is taken. Tersoff potential, one three-body potential based on the concept of bond order, is used to describe the interaction between atoms. The Tersoff potential explicitly includes an angular contribution of the force and has been widely used in various applications for III-V elements. The Tersoff potential and its parameters for the carbon, BN atomic systems see $[13,14]$. In the simulations of the local compression the width of two rigid-plates takes $0.74 \mathrm{~nm}$. The interaction between atoms and the rigid plates is described using the L-J potential:

$$
U(r)=4 \varepsilon\left[\left(\frac{\sigma}{r}\right)^{12}-\left(\frac{\sigma}{r}\right)^{6}\right]
$$

where $r$ is the distance between atoms and rigid-plate; the constants $\varepsilon$ and $\sigma$ take $0.440 \mathrm{~kJ}$ and $0.385 \mathrm{~nm} \mathrm{[15],}$ respectively.

In our EMD simulations, based on the Green-Kubo linear response theory, the thermal conductivity has the following formula [16]

$$
\lambda=\frac{1}{K_{B} T^{2} V} \int_{0}^{+\infty}\langle q(t) \cdot q(0)\rangle d t,
$$

where $K_{B}$ is the Boltzmann constant, $T$ the simulative temperature, $V$ the volume of simulated system, that is, the cross-section area $\left(=\pi D^{2} / 4\right)$ multiplying the tube length, $t$ time, and $q$ the effective heat flux.

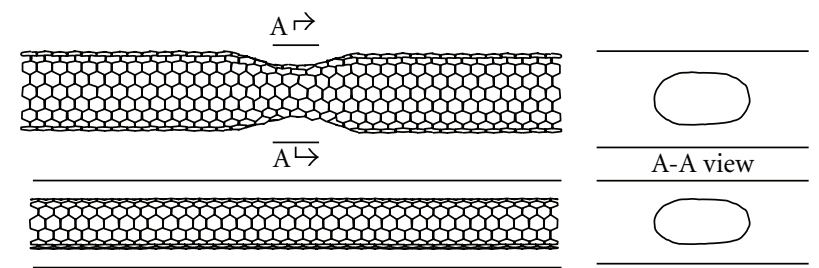

Figure 1: The carbon and BN nanotubes under local and whole radial compression.

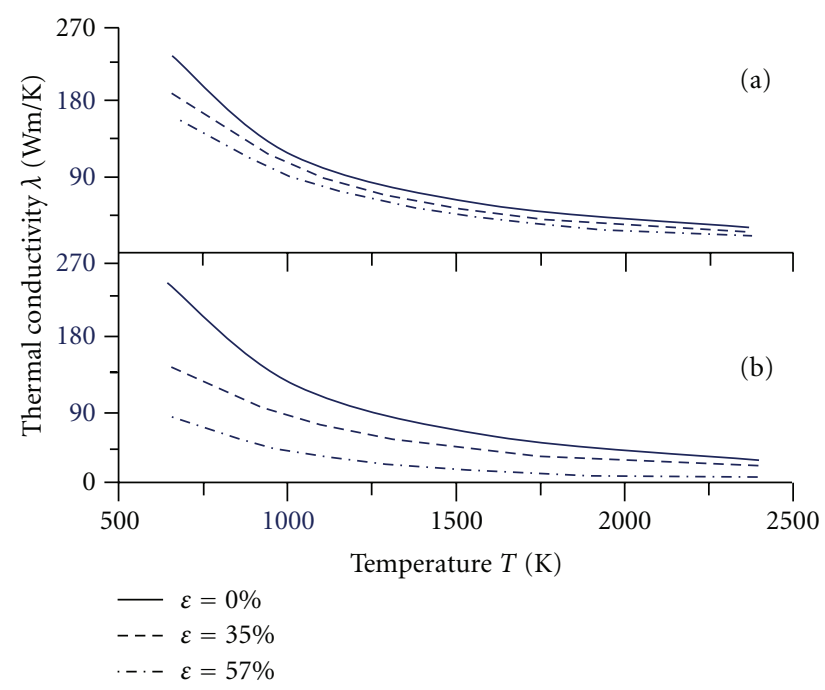

Figure 2: The $\lambda-T$ curves of carbon tube under (a) local and (b) whole compression. of:

The effective heat flux $q$ has the following expression [11]

$$
q(t)=\frac{d}{d t} \sum_{i} \vec{r}_{i}(t) e_{i}(t), \text { that is, } q(t)=\sum_{i} \vec{v}_{i} e_{i}+\sum_{i} \vec{r}_{i} \frac{d e_{i}}{d t},
$$

where $v_{i}$ is the velocity of the $i$ th atom, $e_{i}$ the total energy of the $i$ th atom, and $\bar{r}_{i}$ the coordinate.

\section{Results and Discussion}

It is proved that the contribution to thermal conductivity of nanotube mainly comes from two parts, that is, (1) the interaction between electrons and phonons, that is dependent on electronic structures (molecular configuration) and phonon scattering, and (2) the interaction between phonons, that is, correlative to atomic vibration.

Figure 2 gives the $\lambda-T$ (thermal conductivity versus temperature) curves of the carbon nanotube under different local compressive strain and whole compressive strain $\varepsilon$, and Figure 3 gives those of the BN nanotube. For convenience to discuss, Figure 4 presents the $\lambda$ - $T$ curves of the carbon nanotube under different compression modes, that is, the local compression and whole compression, and Figure 5 presents those of the BN nanotube. In these figures the compressive 


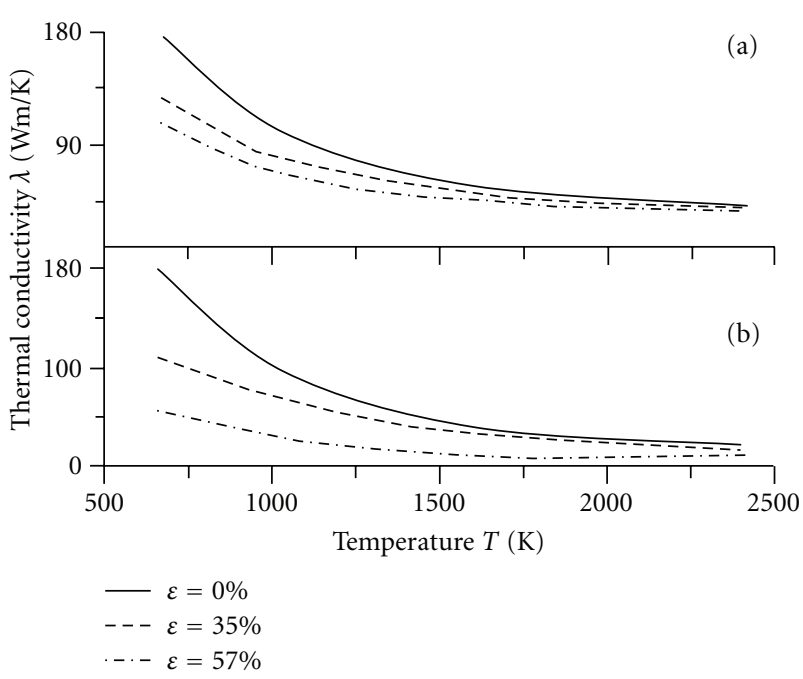

Figure 3: The $\lambda$-T curves of BN tube under (a) local and (b) whole compression.

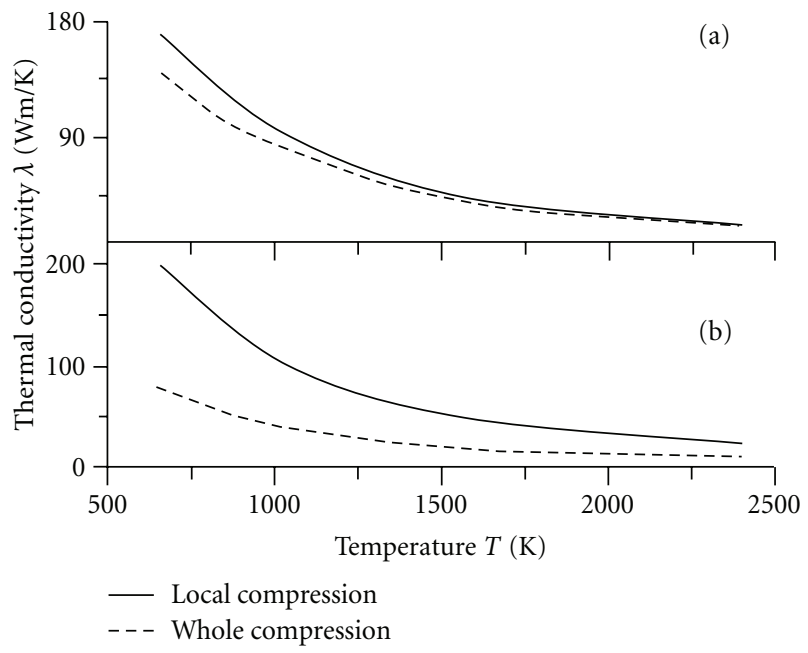

FIgURE 4: The $\lambda$-T curves of carbon tube under different compression mode of (a) $\varepsilon=35 \%$ and (b) $\varepsilon=57 \%$.

strain $\varepsilon$ is defined as the ratio of the radial compressive deformation to the initial tube diameter $D$.

From Figures 2 and 3, it can be seen that,

(1) with the increase of temperature, the thermal conductivity of both the carbon and $\mathrm{BN}$ nanotubes decreases, and when temperature $>1,800 \mathrm{~K}$, their thermal conductivity trends to be constant. What is the reason? It can be explained as the following: due to the increase of temperature, the U scattering process of phonons increases, and the average free range of phonons decreases, which causes the decreases in thermal conductivity of both the carbon and $\mathrm{BN}$ nanotubes;

(2) under the local compression, both the carbon and $\mathrm{BN}$ nanotubes have lower thermal conductivity; the

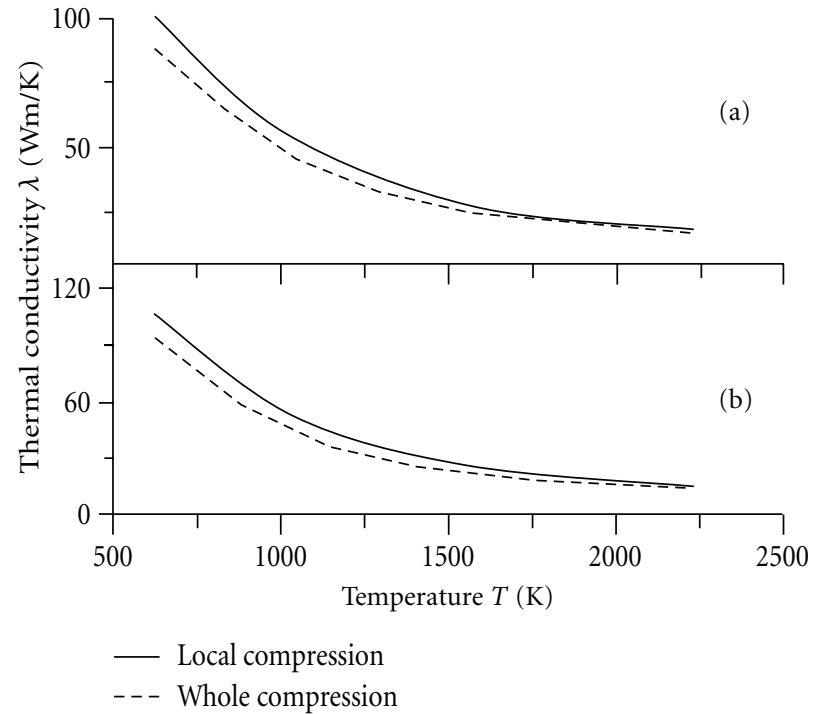

FIgURE 5: The $\lambda$-T curves of BN tube under different compression mode of (a) $\varepsilon=35 \%$ and (b) $\varepsilon=57 \%$.

larger the compressive strain (deformation) is, the lower the thermal conductivity is. The phenomenon can be explained as the following: firstly, the local compression causes local cave-in on the tube wall, that is, the change in local molecular structure, and further the change of the local atoms in vibration mode; secondly, the local cave-in on the tube wall changes the heat exchange path and increases the local phonon U scattering. In a word, the local radial compression makes a bottleneck of heat exchange on the nanotube wall; the larger the compressive deformation is, the worse the thermal conductivity becomes;

(3) at the same temperature, the whole compression also makes the thermal conductivity of both the nanotubes decrease; the larger the compressive deformation is, the lower the thermal conductivity is. The reason lies in that under whole compression the chemical-bonds are tightened up, and the larger the compressive deformation is, the tighter the chemicalbonds become, which results in the change of atoms in vibration mode, that is, vibration amplitude and frequency, as well as the decrease in thermal conductivity;

(4) at relatively low temperature, the effect of both the local and whole compression on the thermal conductivity of the carbon and $\mathrm{BN}$ nanotubes is remarkable. At high temperature, the effect becomes slight, which is because that the effect of compressive deformation has been inundated by that of high temperature.

According to Figures 4 and 5, it can be observed that, under the same temperature and compressive-deformation, the wholly compressed carbon (or BN) nanotube has lower 
thermal conductivity than the locally compressed one. The phenomenon can be explained through the different heattransfer mechanism of the nanotubes under different compression modes, that is, the tightened up chemical-bonds in the wholly compressed nanotubes results in the change of atom vibration mode as well as the change of thermal conductivity; the bottleneck of heat exchange on the tube wall decreases the thermal conductivity of the locally compressed nanotubes.

The present $(10,10)$ carbon nanotube without compressive deformation is predicted to have the thermal conductivity of $180 \mathrm{~W} / \mathrm{mK}$ and $94 \mathrm{~W} / \mathrm{mK}$, respectively, at $800 \mathrm{~K}$ and $1200 \mathrm{~K}$. The corresponding MD results obtained in [17] are $190 \mathrm{~W} / \mathrm{mK}$ and $120 \mathrm{~W} / \mathrm{mK}$, respectively. Our results are close to those of [17], which implies the effectivity of the MD method in the present paper. In $[18,19]$, the measurement of the thermal conductivity of single-layer graphenes was reported, and the room temperature values of the thermal conductivity were found to be in the range 3000 to $5300 \mathrm{~W} / \mathrm{mK}$. Conceptually, single-wall carbon nanotubes can be considered to be formed by the rolling of a single-layer of graphite/graphene. By the cubic polynomial interpolation of the curves in Figure 2, it is found that the thermal conductivity of the $(10,10)$ carbon nanotube without deformation is about $430 \mathrm{~W} / \mathrm{mK}$ at $300 \mathrm{~K}$, lower by one order of magnitude than that of graphenes in $[18,19]$.

\section{Conclusions}

As the important components of future NEMSs (nanomechanical systems), one-dimensional nano-materials like carbon and $\mathrm{BN}$ nanotubes have broad application prospect, and their thermal conductivity and thermal dispersion will directly affect the system operation. In this study the EMD method is used to investigate the effect of local and whole radial compression on the thermal conductivity of carbon and $\mathrm{BN}$ nanotubes. It is found that both the local and whole compression can decrease the thermal conductivity of nanotubes. The conclusion will be very helpful for the design of carbon and $\mathrm{BN}$ nanotube thermal-conductivity-devices.

\section{References}

[1] A. Balandin and K. L. Wang, "Significant decrease of the lattice thermal conductivity due to phonon confinement in a freestanding semiconductor quantum well," Physical Review B, vol. 58, no. 3, pp. 1544-1549, 1998.

[2] N. G. Chopra, R. J. Luyken, K. Cherrey et al., "Boron nitride nanotubes," Science, vol. 269, no. 5226, pp. 966-967, 1995.

[3] A. A. Balandin, "Thermal properties of graphene and nanostructured carbon materials," Nature Materials, vol. 10, no. 8, pp. 569-581, 2011.

[4] J. Hone, M. Whitney, C. Piskoti, and A. Zettl, "Thermal conductivity of single-walled carbon nanotubes," Physical Review B, vol. 59, no. 4, pp. R2514-R2516, 1999.

[5] C. W. Chang, W. Q. Han, and A. Zettl, "Thermal conductivity of B-C-N and BN nanotubes," Applied Physics Letters, vol. 86, no. 17, Article ID 173102, 3 pages, 2005.

[6] Z. Xu and M. J. Buehler, "Strain controlled thermomutability of single-walled carbon nanotubes," Nanotechnology, vol. 20, no. 18, Article ID 185701, 2009.
[7] K. Bi, Y. Chen, J. Yang, and M. Chen, "Molecular dynamics simulation of thermal conductivity of single-wall carbon nanotubes with different structures," Journal of Southeast University, vol. 36, no. 3, pp. 420-422, 2006.

[8] H. Shen, "Generating carbon nanotube and researching into its mechanics properties by HyperChem," Computers and Applied Chemistry, vol. 21, no. 4, pp. 485-488, 2004.

[9] S. Tian and H. Shen, "Thermal and tensile properties of BN, $\mathrm{SiC}$ and Ge nanotubes," Journal of Materials Engineering \& Science, vol. 26, no. 6, pp. 135-139, 2008.

[10] D. Lu and P. Jiang, Solid Physics, Shanghai Science Press, Shanghai, China, 2003.

[11] L. Museur, D. Anglos, J. P. Petitet, J. P. Michel, and A. V. Kanaev, "Photoluminescence of hexagonal boron nitride: effect of surface oxidation under UV-laser irradiation," Journal of Luminescence, vol. 127, no. 2, pp. 595-600, 2007.

[12] M. P. Allen and D. J. Tildesley, Computer Simulation of Liquids, Clarendon Press, Oxford, UK, 1987.

[13] R. Pässler, "Basic moments of phonon density of states spectra and characteristic phonon temperatures of group IV, III-V, and II-VI materials," Journal of Applied Physics, vol. 101, no. 9, Article ID 093513, 2007.

[14] J. Tersoff, "Empirical interatomic potential for carbon, with applications to amorphous carbon," Physical Review Letters, vol. 61 , no. 25 , pp. 2879-2882, 1988.

[15] Y. Chen and H. Shen, "MD simulations of diametrally compressed single-walled and double-walled carbon nanotubes," Journal of Atomic and Molecular Physics, vol. 31, pp. 352-356, 2007.

[16] J. H. Irving and J. G. Kirkwood, "The statistical mechanical theory of transport processes. IV. The equations of hydrodynamics," The Journal of Chemical Physics, vol. 18, no. 6, pp. 817-829, 1950.

[17] H. Chen, Y. Chen, M. Chen, and K. Bi, "Thermal conductivity of single-wall carbon nanotubes filled with argon," Journal of Southeast University, vol. 36, no. 3, pp. 416-419, 2006.

[18] A. A. Balandin, S. Ghosh, W. Bao et al., "Superior thermal conductivity of single-layer graphene," Nano Letters, vol. 8, no. 3, pp. 902-907, 2008.

[19] S. Ghosh, D. L. Nika, E. P. Pokatilov, and A. A. Balandin, "Heat conduction in graphene: experimental study and theoretical interpretation," New Journal of Physics, vol. 11, Article ID 095012, 2009. 

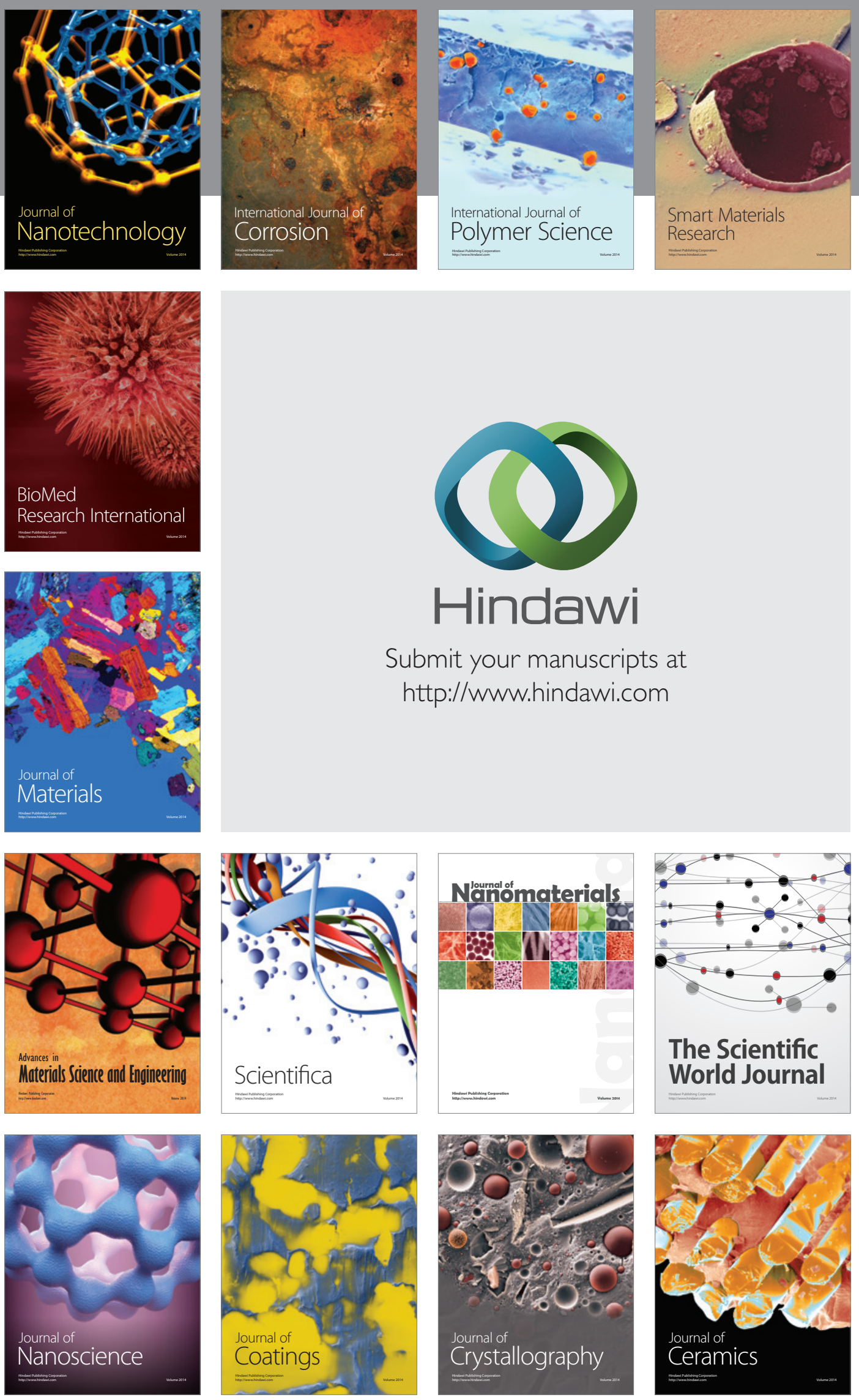

The Scientific World Journal

Submit your manuscripts at

http://www.hindawi.com

\section{World Journal}

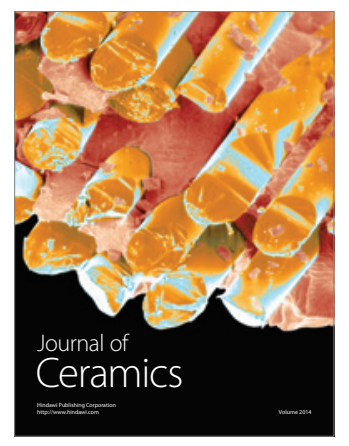

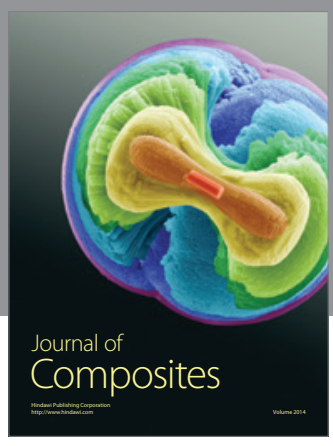
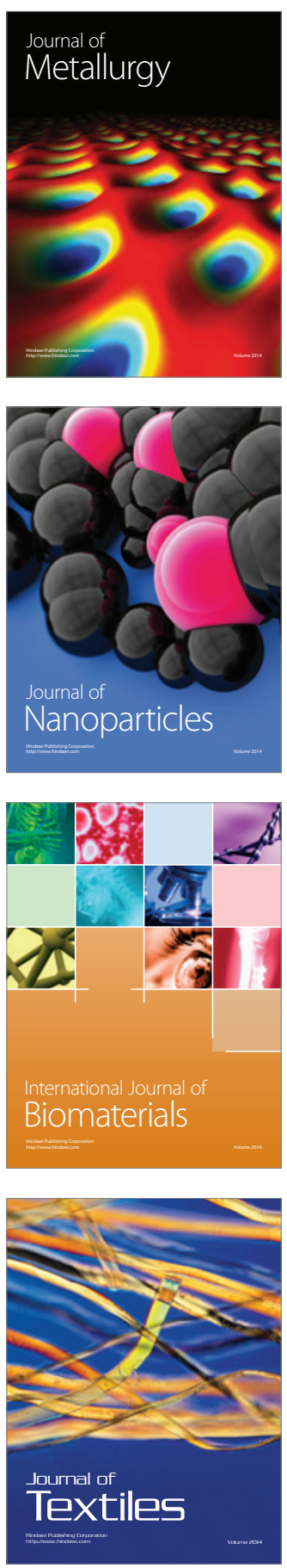